\title{
Gestion des déchets d'équipements électriques et électroniques (DEEE) au Sénégal : acteurs et stratégie d'organisation de la filière
}

\author{
Diomaye DIENG $^{1 *}$, Cheikh DIOP ${ }^{1}$, El hadji Mamadou SONKO ${ }^{1,2}$, Jean Birane GNING ${ }^{1,2,}$ \\ Mamané DJITTE $^{1}$ et Cheikhou Ibrahima Diaby GASSAMA ${ }^{3}$ \\ ${ }^{I}$ Institut des Sciences de l'Environnement, Faculté des Sciences et Technique, \\ Université Cheikh Anta Diop de Dakar. B.P. 5005 Dakar-Fann, Sénégal. \\ ${ }^{2}$ EAWAG: Swiss Federal Institute of Aquatic Science and Technology, \\ Eawag Überlandstrasse 133 P.O.Box 611, 8600 Dübendorf, Switzerland. \\ ${ }^{3}$ Cellule de Solidarité Numérique de l'Agence de l'Informatique de l'Etat (ADIE) ; \\ BP: 6944 Dakar-Etoile ; Sénégal. \\ *Auteur correspondant; E-mail: diengdiomaye@yahoo.fr ; Tel: (00221) 775704999
}

\section{RÉSUMÉ}

La gestion des Déchets d'Equipements Electriques et Electroniques (DEEE) est un défi pour le Sénégal. Leur quantité augmente rapidement et leur gestion informelle expose l'environnement et les acteurs aux risques liés aux substances toxiques qu'ils contiennent. L'étude vise à déterminer les caractéristiques des acteurs impliqués dans la gestion des DEEE et leur connaissance des dangers des composants ; à identifier les circuits et à proposer un schéma de gestion approprié. La méthodologie regroupe la revue de la littérature, des entretiens et des visites de terrains. Les résultats montrent que $80,5 \%$ des importateurs/distributeurs et $63 \%$ des utilisateurs professionnels ont moins de 40 ans; $65,9 \%$ des réparateurs sont âgés au plus de 36 ans. Les acteurs de sexe masculin dépassent $70 \%$ des effectifs interviewés. Par ailleurs, $66,4 \%$ des distributeurs, $67,3 \%$ des utilisateurs professionnels, $59,4 \%$ des ménages et $100 \%$ des groupes composés des recycleurs, récupérateurs et réparateurs ignorent les dangers liés aux composants toxiques des e-déchets. La gestion informelle de ces déchets ne permet pas de tirer profit de tous leurs composants précieux et de créer des richesses et des emplois. Les populations et l'environnement restent exposés aux dangers des e-déchets. L'étude propose des mécanismes adéquats de gestion et de valorisation des DEEE pour encadrer le développement numérique et lutter contre le chômage et la pollution.

(C) 2017 International Formulae Group. All rights reserved.

Mots clés : Gestion, déchets, équipements électriques et électroniques, environnement, composants toxiques.

\section{Management of electrical and electronical equipment wastes (MEEEW) in Senegal: actors and organizational strategy of the sector}

\begin{abstract}
The management of electrical and electronical equipment wastes (MEEEW) is a challenge for Senegal. Their quantity increases quickly and their informal management exposes both the environment and the actors to the risks linked to the toxic substances they contain. This study aimed at determining the characteristics of
\end{abstract}


implicated actors in the (MEEEW) and their knowledge of the danger of the different components; to identify the chains and to propose an appropriate managing plan. This methodology gathers the literature review, maintenances and grounds visits. The results show that $80.5 \%$ of importers / distributors and $63 \%$ professional users have less than 40 years; $65.9 \%$ of repairers are all the more aged of 36 years. The number of men actors exceed of $70 \%$ the number of interviewed. Besides, $66.4 \%$ of distributers, $67.3 \%$ professional users, $59.4 \%$ households and $100 \%$ of groups composed of transformers, recuperators and repairers ignore the danger linked to the toxic substances of e- wastes. The informal management of these wastes does not allow to get interest of all their precious components and to create resources and employments. Both people and the environment are exposed to the dangers of the e-wastes. The study proposes appropriate mechanisms of management and valorization of (EEEW) to control the numeric development and to fight against unemployment and pollution. (C) 2017 International Formulae Group. All rights reserved.

Keywords: management, wastes, electrical and electronical equipment wastes, environment, toxic components.

\section{INTRODUCTION}

Les équipements électriques et électroniques sont aujourd'hui des outils de travail indispensables dans plusieurs secteurs d'activités dans le monde. Ces équipements fonctionnent grâce à un courant électrique ou à un champ électromagnétique, et peuvent servir pour la production, le transfert ou la mesure des courants et champs. Ils sont conçus pour être utilisés à une tension ne dépassant pas 1000 Volts en courant alternatif ou 1500 Volts en courant continu (Directives 2002/96/CE, 2003). En fin de vie, ils deviennent des Déchets d'Equipements Electriques et Electroniques (DEEE) ou déchets électroniques. Chaque année, 20 à 50 millions de tonnes de DEEE sont produites dans le monde (UNEP, 2009). Ce volume est en croissance rapide du fait des fréquences de renouvellement élevées, des modes d'utilisation et de l'innovation technologique favorisant l'obsolescence rapide (Kiddee et al., 2013). En Europe, les DEEE augmentent de trois à cinq pour cent par an, soit près de trois fois plus vite que le flux total de déchets (Kong et al., 2012). En Asie, particulièrement en Chine et en Inde les quantités de DEEE augmentent aussi rapidement d'année en année du fait de la production intérieure mais aussi des importations illégales (Widmer et al., 2005). Dans le cadre de l'initiative StEP (Solving the E-Waste Problem : résoudre le problème des e-déchets), le PNUE montre que la Chine est devenue la deuxième productrice de déchets électroniques au monde, avec 2,3 millions de tonnes par an, derrière les EtatsUnis qui en produisent 3 millions de tonnes (UNEP, 2009). En Afrique, il manque des données consolidées sur ce nouveau phénomène. Toutefois, le diagnostic dans certains pays montre une augmentation des DEEE du fait des systèmes de coopération et des donations en provenance des pays développés. Contrairement aux pays industrialisés qui mettent en place des filières de gestion des DEEE économiquement viables pour toutes les catégories d'équipements, la filière de recyclage dans les pays africains est largement dominée par le secteur informel, qui s'intéresse uniquement à la fraction des déchets présentant une valeur économique sur leur marché (secrétariat de la convention de Bâle, 2011).

Au Sénégal, la lutte contre la fracture numérique engagée depuis le sommet de l'information à Genève, en 2003, a entraîné l'augmentation d'équipements électriques et électroniques. Ces équipements souvent de seconde main deviennent rapidement obsolètes et augmentent ainsi les quantités de DEEE. En 2007, la quantité de déchets issus des ordinateurs et des téléphones portables était de l'ordre de 650 tonnes (Wone et Rochât, 2009). Selon ces auteurs, ce taux devrait dépasser les 2000 tonnes en 2015. Cette quantité, bien qu'étant révélatrice de la croissance des volumes de déchets électroniques, pourrait être sous-estimée du 
fait des stocks non déclarés dans les ménages, les magasins des services de l'administration et des entreprises, les ateliers des réparateurs et des recycleurs, mais surtout des importations frauduleuses de matériels électroniques. En plus des stocks non déclarés, la quantité de DEEE peut connaître des augmentations annuelles importantes car, selon Brett (2009), le nombre d'équipements électroniques est fortement corrélé au Produit Intérieur Brut (PIB) du fait de l'utilisation des équipements de tout genre pour entraîner la croissance des secteurs moteurs de la croissance. $\mathrm{Au}$ Sénégal, la croissance économique estimée à $6,4 \%$ en 2015 , selon le Ministère des finances, se traduira alors par une augmentation de sa production de déchets électroniques.

Ces déchets contiennent à la fois des composants toxiques (plomb, mercure, cadmium) et des matériaux de valeur tels que le plastique, des métaux de base comme l'acier, l'aluminium ou le cuivre, et de métaux précieux comme l'or, l'argent ou le palladium (Secrétariat de la convention de Bâle, 2011). Leur gestion mobilise divers acteurs du secteur informel. Cela pose le problème des circuits et de la traçabilité des déchets durant le processus de gestion. L'étude sur les DEEE de Wone et Rochat (2009) n'est que peu documentée car elle ne concerne que la région de Dakar. Les données collectées n'ont pas permis d'appréhender les interrelations entre les acteurs au niveau national et d'apprécier le niveau de connaissance des populations quant aux risques liés aux DEEE compte tenu des niveaux de développement différents des régions. Diop et Thioune (2014) ont tenté de corriger ces écarts mais le caractère exploratoire de leur étude n'a pas permis de développer les aspects techniques du recyclage et de proposer un plan de gestion durable des DEEE.

Les faiblesses des études précédentes ont motivé cette présente recherche qui a comme objectifs de déterminer le profil des acteurs impliqués dans la gestion des déchets électroniques, d'analyser les circuits actuels desdits déchets et de proposer un schéma inclusif de leur gestion.

\section{MATÉRIEL ET MÉTHODES \\ Cadre de l'étude}

Cette étude est réalisée au Sénégal. Pays situé à l'extrême Ouest du continent africain, entre $12^{\circ} 5$ et $16^{\circ} 5$ de latitude Nord et $11^{\circ} 5$ et $17^{\circ} 5$ de longitude Ouest, le Sénégal couvre une superficie de $196712 \mathrm{Km}^{2}$. Il dispose d'une façade maritime longue d'environ $700 \mathrm{~km}$ (CSE, 2010). Le Sénégal est caractérisé par de profondes disparités marquées par les poids démographiques et économiques de l'axe Dakar-Thiès. La région de Dakar concentre $25 \%$ de la population du Sénégal (environ 3 millions) sur une superficie de $0,3 \%$ du territoire national et plus de $80 \%$ des industries. En 2013, la population du Sénégal était estimée à 13508 715 habitants, soit une densité de 68,67 habitants au $\mathrm{Km}^{2}$ (ANSD, 2013). Au Sénégal, pour l'année 2013, la croissance du PIB réel a été de $3,5 \%$; près de $20 \%$ de la population active sont au chômage et le taux de pauvreté était estimé à 46,7 \% en 2011 (Banque Mondiale, 2014). Les TIC contribuent à hauteur de $6 \%$ du PIB sur une contribution globale des transports, postes et télécommunication de l'ordre de 10,8\% (ANSD, 2006). Les informations sur le niveau de développement du Sénégal expliquent sa dépendance vis-à-vis des pays développés pour l'acquisition de matériels électriques et électroniques. Le secteur informel occupe une part importante des activités commerciales et économiques du pays. L'enquête sur les DEEE a concerné onze (11) régions les plus anciennes sur les quatorze (14) que compte le pays. Ce choix est basé aussi selon le niveau de développement et les activités socioéconomiques qui s'y déroulent.

\section{Collecte de données}

La collecte des données est faite à travers la revue de la littérature pour identifier et catégoriser les acteurs, complétée par des visites de terrain et l'application de questionnaires et de guides d'entretien aux 
acteurs impliqués dans la gestion des DEEE. Les acteurs identifiés sont composés des distributeurs, des importateurs, des utilisateurs professionnels, des ménages, des récupérateurs, des réparateurs et des recycleurs. L'échantillonnage auprès de chaque catégorie d'acteur est effectué sur la base d'un choix raisonné.

$\mathrm{Au}$ niveau des distributeurs et des importateurs, un effectif global de 159 individus a été enquêté. Un même questionnaire est administré aux deux catégories d'acteurs du fait de leurs actions souvent imbriquées. En effet, certains distributeurs s'adonnent dans bien des cas à l'importation de matériels électroniques et d'autres importateurs procèdent également à la vente.

$\mathrm{Au}$ niveau des utilisateurs, nous avons distingué les utilisateurs professionnels et les ménages. Parmi les utilisateurs professionnels, 274 individus des services de l'administration (28,1\%), des sociétés privées (opérateurs de téléphonie, banques, entreprises, etc.) $(18,7 \%)$, des écoles $(12,8 \%)$, des cybercafés $(31,5 \%)$, des particuliers $(60 \%)$ et d'autres catégories non spécifiées $(30 \%)$ ont été enquêtés. Pour les ménages, 1504 chefs de ménages ont été choisis et enquêtés parmi les 1296200 ménages que compte le Sénégal, soit $0,116 \%$ de l'effectif total (ANSD, 2006).

$\mathrm{Au}$ niveau des acteurs de l'économie solidaire, le questionnaire a été administré à 43 recycleurs et à 44 réparateurs. Du fait des rôles souvent imbriqués des réparateurs et des recycleurs, il a été difficile de les classer. Nous nous sommes basés sur les opérations qui dominent dans leurs ateliers pour les catégoriser. Des focus groupe ont été effectués avec des récupérateurs évoluant dans la décharge de Mbeubeuss (plus grande décharge à ciel ouvert de Dakar) du fait qu'ils constituent un groupe quasi-homogène de par leur zone d'intervention et leurs activités. Un guide d'entretien a été appliqué aux récupérateurs.

\section{RÉSULTATS \\ Profil des acteurs}

L'analyse du profil des acteurs s'est intéressée à l'âge, au sexe, au niveau d'instruction, à la connaissance du danger associé aux composants des DEEE et aux modes de gestion des DEEE. Le choix de ces paramètres est lié à leur influence sur la gestion des DEEE, car Diop et Thioune (2014) ont montré qu'un taux de chômage élevé et un alphabétisme faible agissent sur la perception et les modes de gestion des DEEE.

Les résultats selon l'âge montrent une prédominance des jeunes (avec plus de 50\%) dans toutes les catégories. A titre d'exemple, les moins de 40 ans représentent respectivement $80,5 \%$ des importateurs/ distributeurs et $63 \%$ des utilisateurs professionnels. Au niveau des ménages, les équipements électriques et électroniques sont utilisés en majorité par les jeunes qui sont plus sensibles à la mode et à l'évolution des technologies. Plus de la moitié des recycleurs $(57,1 \%)$ et $65,9 \%$ des réparateurs sont âgés au plus de 36 ans. La récupération est un secteur très dynamique où on trouve pratiquement toutes les tranches d'âge, les femmes et les enfants sont très présents car souvent c'est une affaire de famille.

Selon le sexe, il y a une prédominance des hommes par rapport aux femmes. Les individus de sexe masculin représentent respectivement $91,8 \% ; 88 \% ; 72,5 \%$; $97,7 \%$ et $100 \%$ des effectifs des importateurs/ distributeurs, des utilisateurs professionnels, des ménages, des réparateurs et des recycleurs.

Selon le niveau d'instruction des acteurs, les personnes n'ayant pas un niveau d'instruction représentent respectivement $5 \%$; $0 \% ; 6,1 \% ; 2,3 \%$ et $0 \%$ des importateurs/ distributeurs, des utilisateurs professionnels, des ménages, des réparateurs et des recycleurs. Parmi les personnes instruites, on distingue celles qui sont alphabétisées en arabe et celles alphabétisées en français. Les populations alphabétisées en arabes constituent la proportion la plus importante parmi les importateurs/distributeurs (33\%). 
Elles représentent respectivement 2,3\% ; $14 \% ; 13,7 \%$ et $18,6 \%$ des utilisateurs professionnels, des ménages, des réparateurs et des recycleurs. Parmi les personnes alphabétisées en français, celles ayant un niveau supérieur prédominent au niveau des utilisateurs professionnels (53\%) alors qu'elles représentent respectivement $12 \%$; $28,6 \% ; 15,9 \%$ et $9,3 \%$ des utilisateurs/ distributeurs, des ménages, des réparateurs et des recycleurs. Le niveau moyen-secondaire est plus représenté chez les réparateurs $(54,5 \%) \quad$ contrairement aux importateurs/distributeurs $\quad(32,7 \%), \quad$ les utilisateurs professionnels (33\%), les ménages $(4 \%)$ et les recycleurs $(32,6 \%)$. Le niveau primaire est plus représenté chez les recycleurs avec $39,5 \%$ contre respectivement $16 \% ; 11,3 \% ; 16,9 \%$ et $13,6 \%$ chez les importateurs/distributeurs, les utilisateurs professionnels, les ménages et les réparateurs.

La connaissance des dangers associés à la gestion des DEEE est très faible indépendamment du niveau d'instruction. Chez les utilisateurs professionnels, constitués à $53,2 \%$ d'individus ayant un niveau supérieur d'instruction, la méconnaissance du danger demeure la plus importante avec une proportion $67,3 \%$ des acteurs ignorant les dangers associés aux DEEE. Chez les importateurs et distributeurs ce taux reste également élevé avec $66,4 \%$ d'individus ignorant les dangers liés à ce type de déchet. Pourtant, parmi ces acteurs, $44,7 \%$ ont au moins le niveau moyen secondaire et $33,3 \%$ sont alphabétisés en arabe.

Le Tableau 2 montre la variation du niveau de connaissance des dangers des DEEE par les ménages et selon la région. Il révèle que plus de la moitié des ménages $(59,4 \%)$ n'a pas de connaissances sur les conséquences négatives des DEEE sur la santé et l'environnement. A Dakar, $62,9 \%$ des ménages enquêtés ignorent les effets négatifs de ces déchets.

Les récupérateurs qui ne font que transférer les DEEE d'un acteur à un autre n'ont pas de connaissance sur les dangers des composants toxiques des DEEE. Cependant, les blessures sont évoquées comme risques chez les recycleurs (31\%). Au niveau de ce groupe également, même si les acteurs ignorent la toxicité des composants des edéchets, du fait de leur statut de produits en fin de vie, ils précisent, entre autres, les risques des maladies $(13 \%)$, de dégradation de l'environnement $(15 \%)$, de nuisances et de pollutions (13\%), ainsi que la dépréciation du marché des sous-produits récupérés (28\%).

Dans une dynamique de promouvoir la responsabilité élargie du producteur, cette étude a permis d'apprécier la possibilité de reprise des équipements en fin de vie par les distributeurs. Cependant, selon le Tableau 1, seulement $48,7 \%$ en moyenne adhèrent à la reprise.

Par ailleurs, au niveau des ménages, le versement d'une contribution financière pour la prise en charge des DEEE a été étudié. Le Tableau 3 montre que plus de la moitié des ménages $(55,4 \%)$ accepte de verser une cotisation à cet effet. Toutefois, les données du Tableau 3 précisent que les acteurs des grandes villes, Dakar et Thiès adhèrent plus à cette option, avec respectivement $61,2 \%$ et $61,7 \%$, à l'exception de Kaolack $(85,6 \%)$.

Le Tableau 4 présente l'engagement des utilisateurs professionnels à contribuer à la gestion des DEEE à travers le choix du type de matériel, une participation financière et/ou une bonne sensibilisation des acteurs.

Les résultats montrent que le type d'utilisateur, la connaissance de la toxicité des composants des DEEE, la région d'intervention, les capacités financières et techniques des acteurs influent sur les modes de gestion des DEEE.

\section{Circuit informel actuel des DEEE}

Les interrelations entre les différents acteurs, illustrées par la Figure 1, montrent un manque d'organisation dans la gestion des DEEE. Au Sénégal, les importateurs/distributeurs peuvent être considérés comme la source initiale de production des DEEE. Ils alimentent les différents acteurs en appareils électroniques. Plus de la moitié (55\%) d'entre eux affirme 
que leur clientèle est composée de fonctionnaires; contre $43 \%, 43 \%, 42 \%$ et $42 \%$ qui vendent leurs équipements respectivement aux étudiants/ménages, aux sociétés privées, aux services de l'administration et aux entreprises publiques. Outre les appareils électroniques en bon état, ils mettent dans le circuit les appareils obsolètes participant ainsi de façon directe à la production des DEEE. Les déchets électroniques sont, en effet, jetés dans les poubelles ou les décharges sauvages d'ordures ménagères $(16 \%)$, incinérés $(1 \%)$, ou livrés aux recycleurs (30\%), ou stockés dans les magasin (53\%) en attendant de trouver une solution.

$\mathrm{Au}$ niveau des utilisateurs professionnels et des ménages, les équipements hors d'usage sont soit donnés aux récupérateurs, ou aux réparateurs, soit jetés dans les ordures ménagères. Les utilisateurs constituent les principaux partenaires des réparateurs. Un taux de 94,9\% de ces acteurs reçoit les articles venant des utilisateurs. Certains équipements sont restitués aux propriétaires après la réparation alors que d'autres sont soit stockés de façon sauvage dans les ateliers des réparateurs, soit jetés ou même vendus aux autres acteurs (recycleurs et récupérateurs) pour servir de matières premières.

Une partie des équipements fournis aux réparateurs provient des récupérateurs $(15,4 \%$ des équipements récupérés). Le travail des récupérateurs se limite, le plus souvent, à la collecte et à la commercialisation des produits issus des déchets. Etant au bout du circuit, les récupérateurs et les recycleurs procèdent principalement au démantèlement et à l'assemblage de composants divers pour remettre dans le circuit un équipement ou des matériaux recyclés au profit des ménages. Par ailleurs, pour ces acteurs, la décharge constitue un gisement important de matières récupérables capables de leur fournir des revenus substantiels, à l'image des populations de Ouessé (Benin), (DONOU, 2016). Ils remettent dans le circuit de 1'utilisation les équipements recyclés et jettent les parties qu'ils ne peuvent pas valoriser.

La Figure 1 traduit le niveau d'intervention des acteurs et leurs interrelations. Elle montre également que la gestion actuelle des déchets électroniques au Sénégal est informelle et mobilise divers acteurs.

Tableau 1 : Répartition des distributeurs selon la zone et l'acceptation de reprendre les DEEE.

\begin{tabular}{lccc}
\hline Régions & Oui & Non & TOTAL \\
\hline Dakar & 48,3 & 51,7 & 100 \\
Thiès & 29,4 & 70,6 & 100 \\
Louga-St Louis-Diourbel & 39,1 & 60,9 & 100 \\
Fatick-Kaolack & 91,7 & 8,3 & 100 \\
Kédougou-Kolda-Tambacounda & 57,9 & 42,1 & 100 \\
Ziguinchor & 70,0 & 30 & 100 \\
TOTAL & $\mathbf{4 8 , 7}$ & $\mathbf{5 1 , 3}$ & $\mathbf{1 0 0}$ \\
\hline
\end{tabular}


Tableau 2 : Connaissance des dangers liés au DEEE par les utilisateurs (ménages) et selon les régions.

\begin{tabular}{llll}
\hline Régions & Oui & Non & Total \\
\hline Dakar & 37,1 & 62,9 & 100,0 \\
Thiès & 55,7 & 44,3 & 100,0 \\
Louga & 19,7 & 80,3 & 100,0 \\
Saint Louis & 38,2 & 61,8 & 100,0 \\
Diourbel & 41,2 & 58,8 & 100,0 \\
Fatick & 43,1 & 56,9 & 100,0 \\
Kaolack & 39,0 & 61,0 & 100,0 \\
Kolda & 20,5 & 79,5 & 100,0 \\
Tambacounda & 36,7 & 63,3 & 100,0 \\
Ziguinchor & 60,0 & 40,0 & 100,0 \\
\hline Total & $\mathbf{4 0 , 6}$ & $\mathbf{5 9 , 4}$ & $\mathbf{1 0 0 , 0}$ \\
\hline
\end{tabular}

Tableau 3 : Pourcentage des ménages prêts à supporter le coût de traitement des DEEE selon les régions.

\begin{tabular}{lccc}
\hline Régions & Oui & Non & Total \\
\hline Dakar & 61,2 & 38,8 & 100,0 \\
Thiès & 61,7 & 38,3 & 100,0 \\
Louga & 50,8 & 49,2 & 100,0 \\
Saint Louis & 42,9 & 57,1 & 100,0 \\
Diourbel & 13,0 & 87,0 & 100,0 \\
Fatick & 0,0 & 100,0 & 100,0 \\
Kaolack & 85,6 & 14,4 & 100,0 \\
Kolda & 19,2 & 80,8 & 100,0 \\
Tambacounda & 28,9 & 71,1 & 100,0 \\
Ziguinchor & 39,2 & 60,8 & 100,0 \\
\hline Total & $\mathbf{5 5 , 4}$ & $\mathbf{4 4 , 6}$ & $\mathbf{1 0 0 , 0}$ \\
\hline
\end{tabular}


Tableau 4 : Répartition des utilisateurs professionnels selon le rôle qu'ils sont prêts à jouer dans la gestion des DEEE.

\begin{tabular}{cccc}
\hline $\begin{array}{c}\text { Type d'Utilisateurs } \\
\text { Professionnels (UP) }\end{array}$ & \multicolumn{2}{c}{$\begin{array}{c}\text { Quelques utilisateurs Professionnels selon les bonnes pratiques et } \\
\text { contributions à la gestion des DEEE }\end{array}$} \\
\cline { 2 - 4 } & $\begin{array}{c}\text { choix de l'Achat des } \\
\text { Matériels neufs en } \\
\text { pourcentage }\end{array}$ & $\begin{array}{c}\text { choix d'une } \\
\text { Participation Financière } \\
\text { en pourcentage }\end{array}$ & $\begin{array}{c}\text { Sensibilisation en } \\
\text { pourcentage }\end{array}$ \\
\hline Administration & 58,1 & 20,9 & 30,2 \\
Société privée & 80 & 13,3 & 16,7 \\
Lycée/école/formation & 84 & 20 & 80 \\
Cyber & 78,6 & 17,9 & 10,7 \\
Particuliers & 100 & 0 & 0 \\
Autres & 83,3 & 16,7 & 0
\end{tabular}

NB : Tableau à réponses multiples, la somme des pourcentages (\%) en ligne peut dépasser 100\%.

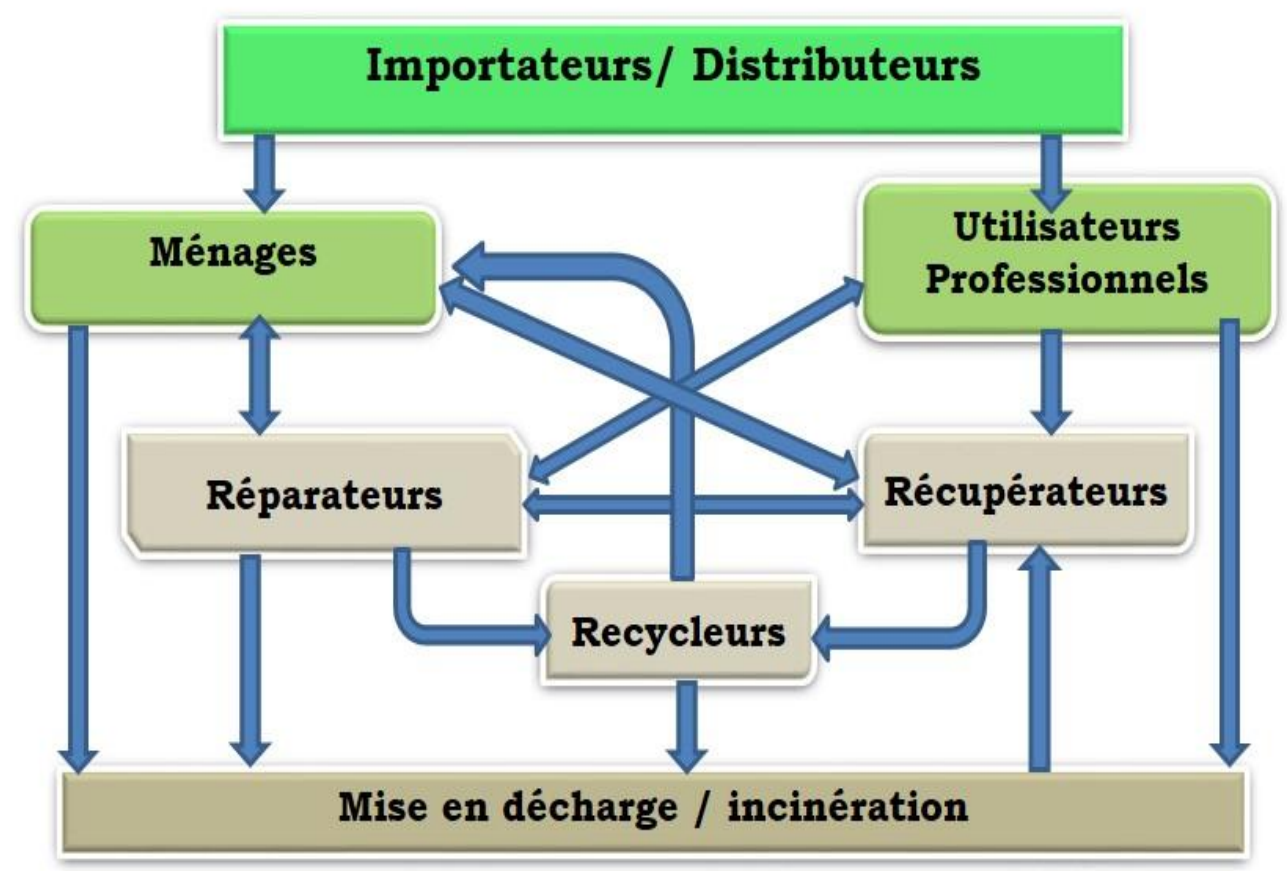

Figure 1 : Circuit informel actuel des DEEE au Sénégal. 


\section{DISCUSSION}

\section{Acteurs et étapes de la chaîne de gestion des DEEE}

Les acteurs intervenant dans la gestion des DEEE sont principalement des jeunes, comme le montre la répartition des classes d'âge au sein de la population sénégalaise caractérisée par sa jeunesse, avec un âge moyen de 22,4 ans, dont la moitié a 18,7 ans (âge médian) (ANSD, 2013). La prédominance des moins de 40 ans s'explique par le taux de chômage élevé des jeunes (plus de $10,2 \%$ ) qui s'orientent vers le secteur informel, selon une étude de la Banque Mondiale (2014). Par ailleurs, les activités comme la réparation, la récupération et le recyclage qui demandent beaucoup d'efforts physiques justifient cette prédominance des jeunes parmi les différents acteurs. Ce même constat est valable pour les acteurs de sexe masculin qui constituent 70 à $100 \%$ des enquêtés selon les catégories. Ce rapport en faveur des hommes s'explique par les efforts physiques que demande la manipulation des équipements électroniques. Il serait lié également à la responsabilisation des hommes dans les entreprises et les ménages mais également à l'émergence du secteur informel qui mobilise les hommes $(57,8 \%)$ et une minorité de femmes (43,1\%) (ANSD, 2009).

Parmi les importateurs/distributeurs, ceux alphabétisées en arabes constituent la portion la plus importante $(33 \%)$. Cela est lié à la présence des «baol-baol» pour lesquels l'enseignement coranique fait partie de l'éducation de base. Ces acteurs sont peu sensibilisés quant aux dangers des DEEE, car, ils sont souvent en contact avec les produits neufs ou qui fonctionnement.

L'utilisation des équipements électriques et électroniques à des fins professionnelles requiert un certain niveau d'instruction au vu des différentes applications. Malgré un niveau d'instruction supérieur en français pour près de $53,2 \%$ des utilisateurs professionnels, une proportion de $67,3 \%$ parmi eux ignore les dangers associés aux DEEE, parce qu'ils ne s'occupent que de l'utilisation des équipements qui, une fois en fin de vie, sont stockés ou livrés aux acteurs $\mathrm{du}$ secteur informel. Les utilisateurs professionnels de même que les ménages ne sont pas en contact directe avec les composants toxiques des déchets électroniques lors de l'utilisation. Ainsi, les dangers desdits déchets sont loin d'être parmi leurs préoccupations majeures.

Pour les acteurs participant au démantèlement manuel et recyclage des déchets électroniques, chaque groupe a une certaine représentation du danger spécifiquement en rapport avec son activité. Ainsi, même s'ils ignorent complètement les risques associés aux composants dangereux des DEEE, les récupérateurs, les réparateurs et les recycleurs soulignent les risques de blessures et d'électrocution. Cependant, le constat est que le système de gestion actuel des DEEE est informel. Il est articulé principalement autour de quatre (4) étapes notamment, le stockage, le recyclage, la mise en décharge et l'incinération. Toutes ces méthodes artisanales sont associées à des conséquences néfastes sur l'environnement et la santé du fait que les déchets électroniques contiennent des composants toxiques comme le plomb, le mercure, le béryllium (EEA, 2003; UNEP, 2009). Ces métaux lourds peuvent avoir divers effets néfastes sur la santé des populations et contaminer les sols et les produits agricoles dans les zones de dépôt des DEEE (Zhao et al., 2008; Wang et al., 2009). Il est établi par exemple que l'anémie est une importante expression de la toxicité du cadmium qui augmente la destruction des globules rouges et diminue leurs synthèses (Diaby et al., 2016).

En 2008, dans un quartier périphérique de Dakar (Thiaroye), l'essor de l'activité de recyclage des batteries à plomb avait entraîné le décès d'au moins dix-huit (18) enfants dans la zone où sur 47 enfants testés, 37 cas nécessitaient un traitement chélateur urgent, soit $68 \%$ (OMS et MEPN, 2008). Par ailleurs, l'incinération DEEE favorise l'émissions de dioxines et de furanes (EEA, 2003 ; UNEP, 2009 ; Chan et al., 2012). Ces substances peuvent conduire à une perturbation du 
développement du système nerveux, des troubles de régulations endocriniennes, des modifications dans la croissance et le développement fondamental des cellules, ce qui peut entraîner des effets indésirables sur la reproduction et le développement ainsi que la destruction du système immunitaire et provoquer le cancer (US EPA, 2012). Malgré ces effets négatifs associés aux DEEE, les données sur la cartographie des zones à risque sont totalement absentes dans les pays africains (Frazzoli et al., 2009). Cette situation nécessitant une attention particulière est en contraste avec la présence de matériaux de valeur tels que le plastique, des métaux de base comme l'acier, l'aluminium ou le cuivre, et de métaux précieux comme l'or, l'argent ou le palladium (Secrétariat de la convention de Bâle, 2011) dont la gestion mobilise divers acteurs du secteur informel.

L'engagement des acteurs à participer à la gestion des DEEE par la reprise des équipements obsolètes, la connaissance des impacts des DEEE sur l'environnement et la santé, la contribution financière et la sensibilisation présentés respectivement à travers les Tableaux 1,2, 3 et 4 sont des gages de réussite d'une bonne prise en charge de ces déchets. Il importe de souligner que les acteurs n'ayant pas un niveau de connaissance élevé par rapport aux dangers des DEEE proposent des réponses multiples en termes de contribution au processus. Pour mieux déterminer leur rôle dans le processus de gestion des déchets électroniques, la connaissance des dangers des composants des DEEE et la promotion de l'achat de produits neufs permettrait le succès d'un plan d'organisation formelle de la gestion desdits déchets.

\section{Proposition d'un plan d'organisation formelle du circuit de gestion des DEEE}

Les résultats de cette étude montrent la nécessité de mettre en place une bonne organisation pour pallier les effets négatifs résultant des DEEE. Selon Qu et al. (2013), chaque pays a besoin d'un système de recyclage qui prend en compte non seulement ses ressources, mais aussi ses réalités économiques et sociales. Sous cet angle, au Sénégal, la Cellule de Solidarité Numérique, l'entreprise SetTIC, l'ONG Eau-VieEnvironnement (EVE) et l'Institut des Sciences de l'Environnement (ISE) de l'Université Cheikh Anta Diop de Dakar ont divers programmes de gestion des DEEE. Malheureusement, ces acteurs ont mené leurs activités de façon isolée, ce qui n'a pas permis d'avoir jusque-là beaucoup l'efficacité souhaitée dans la gestion des déchets. Les limites $\mathrm{du}$ système de gestion actuel s'illustrent aussi par les méthodes peu performantes de collecte, de stockage et de valorisation adoptées par les acteurs.

Aujourd'hui, certains acteurs ont pris conscience des faiblesses et ont décidé de mutualisé leurs efforts pour améliorer le système de gestion et s'orienter vers des solutions qui misent sur la promotion du partenariat public privé (PPP). Un tel système désorganisé de gestion de ces types de déchets a été décrit par Qu et al. (2013) à Dalian en Chine ou de petits groupes de marchands ambulants collectent la plupart des e-déchets en parcourant les communautés pour acheter les appareils ménagers usagés.

La reprise des DEEE par les distributeurs peut garantir l'organisation de la filière et la mise en place d'une plateforme de collecte et de gestion des DEEE. Cependant, il est important de préciser que le pourcentage des acteurs acceptant la reprise des équipement en fin de vie est plus bas dans les grandes villes comme Dakar, Thiès, SaintLouis, Louga et Diourbel où ils sont confrontés à des problèmes d'espace dans leurs locaux qu'ils occupent souvent en tant que locataires. Par ailleurs, ces acteurs des grandes villes comme Dakar $(61,2 \%)$ et Thiès $(61,7 \%)$ adhèrent plus à l'option de faire une contribution financière pour participer à la gestion des DEEE. Le taux record de Kaolack $(85,6 \%)$ est lié aux initiatives privées et à l'effort collectif de la population à lutter contre l'insalubrité dans cette ville longtemps qualifiée comme la «moins propre» au Sénégal (Tableau 3). 
Ainsi, au-delà du diagnostic et de l'identification des acteurs et de leur contribution au processus de gestion des DEEE, cette étude propose d'élaborer un schéma d'organisation formelle de gestion des DEEE, bien encadré au plan institutionnel, selon le modèle de la Figure 2 qui permettra de créer des emplois et de préserver l'environnement.

La Figure 2 propose un circuit articulé autour de quatre étapes. Il s'agit principalement :

- de la collecte des déchets produits au niveau des utilisateurs ;

- du regroupement au niveau d'une plateforme où les équipements seront testés et classés selon la possibilité de leur reconditionnement pour le réemploi ou de leur démantèlement en vue d'une valorisation matière ou énergétique ;

- de la mise en service d'une station de démantèlement pour récupérer les différents composants;

- et de la création d'une station de valorisation et d'élimination contrôlée des DEEE pour leur gestion écologiquement rationnelle.

Pour l'atteinte des objectifs, la connaissance du gisement des déchets électroniques est un préalable pour évaluer la rentabilité d'une unité de recyclage au Sénégal. Une estimation des importations des équipements électroniques dans le pays peut être envisagée comme une étape importante vers l'estimation exhaustive des quantités de DEEE qui y sont produits. Ainsi, selon Wone et Rochât (2009), ce taux peut être défini par l'équation suivante :

\section{$I_{i}=I_{0}(1+x)^{i}$}

Avec $\boldsymbol{I}_{\boldsymbol{0}}$, le flux d'équipements entrants dans le pays à l'année de référence ; $\boldsymbol{I}_{\boldsymbol{i}}$, le flux pour une année donnée et $\boldsymbol{x}$, la tendance géométrique ou coefficient de progression des flux.

Les quantités de DEEE collectées au Sénégal sont très faibles par rapports aux estimations résultant des taux qui entrent dans le pays. Cela pose la problématique des méthodes de quantification des EEE. En effet, avec la croissance du commerce intersubsaharien d'équipements électriques et électroniques, certains équipements transitent d'un pays à un autre. En plus, des lots d'équipements entrent de manière frauduleuse au Sénégal. Ce flux qui échappe au contrôle des services douaniers peut être défini par une variable $K$. Ainsi, en tenant compte de ces paramètres susmentionnés, l'équation précédente s'établit comme suit :

$I_{i}=I_{0}(1+x)^{i}+K_{i}$

Avec $\boldsymbol{K}_{\boldsymbol{i}}$ représentant le flux qui échappe au contrôle douanier.

Selon Enda (2006), ce flux peut atteindre jusqu'à $30 \%$ des quantités de matériel officiellement déclarées par les services chargés du contrôle des importations. L'estimation du gisement des DEEE doit tenir compte de ces paramètres pour être plus ou moins acceptable.

Au regard des chiffres officiels fournis par les services des douanes, la quantification approximative des déchets produits peut être faite à partir des transferts annuels d'une catégorie d'état vers une autre catégorie d'état plus obsolète. Selon Wone et Rôchat (2009), l'équation de bilan du Flux global annuel (F) des déchets produits à partir des Equipements Electriques et Electroniques (EEE) peut être donnée par la formule suivante :

$\boldsymbol{F}=\boldsymbol{F}_{\boldsymbol{o}}+\boldsymbol{F}_{\boldsymbol{n}}+\boldsymbol{F}_{\mathrm{s} \boldsymbol{m}}+\boldsymbol{F}_{\boldsymbol{r}}$

L'estimation des quantités de DEEE relève ainsi de l'addition au flux initial $\mathrm{F}_{0}$ des nouvelles affectations des catégories (neufs, seconde main, récupérés) à laquelle on ajoute

- Celles passant des catégories d'état neuf vers le lot des déchets $\left(\mathrm{F}_{\mathrm{n}}\right)$;

- Celles passant des catégories d'état de seconde main vers le lot des déchets $\left(\mathrm{F}_{\mathrm{sm}}\right)$;

- Celles des catégories des recyclés (pièces et objet) vers les déchets $\left(\mathrm{F}_{\mathrm{r}}\right)$.

L'importance du flux annuel permet de connaître la disponibilité de matières premières pour les acteurs de l'économie circulaire. Car, il est prouvé que les DEEE 
contiennent des éléments tels que le fer et l'acier pour plus de $50 \%$ du poids total des déchets électroniques, les plastiques pour $21 \%$, les métaux non ferreux et les métaux précieux pour $13 \%$ du poids total des déchets électroniques, le caoutchouc, le béton et la céramique pour 16\% (Pant et al., 2012). Une meilleure gestion des déchets de conteneurs plastiques doit passer par une meilleure connaissance des critères de leur choix par les acteurs (Some et al., 2016). Ainsi, une collecte bien organisée permettrait de mieux connaître les gisements des DEEE en vue d'une exploitation sous forme de filières.

Cependant, un système efficace de collecte des déchets électroniques nécessite la mise en place d'infrastructures de soutien comme les centres de collecte et de transfert (Qu et al., 2013). La première étape de la collecte consiste à rassembler le matériel obsolète au niveau de chaque producteur. Cette phase qualifiée de pré-collecte doit être entreprise à l'échelle individuelle comme cela se fait en Allemagne selon Rotter et al. (2011). Les utilisateurs professionnels quant à eux peuvent disposer de points de collecte au sein de leur institution, tandis que les ménages peuvent, après la pré-collecte, transmettre leurs DEEE aux distributeurs, aux réparateurs/recycleurs ou même les mettre dans des poubelles spécifiques installées à cet effet. Une telle organisation de la collecte a été rapportée par Kang et Schoenung (2005) aux USA montrant que les facteurs clés de la gestion des déchets électroniques sont la collecte, le tri et la récupération, le recyclage et l'élimination ; puis par Qu et al. (2013) en Chine où un réseau de coopération entre les institutions publiques, une société privée de traitement des déchets électroniques (Dongtai) et la commune de Dalian (Qu et al., 2013) a été développé. Les contraintes du système de collecte et de recyclage des DEEE sont nombreuses au Sénégal où le dispositif demeure insuffisant et informel. Pour l'organisation de la filière, après la phase de pré-collecte effectuée par les utilisateurs professionnels et les ménages, un opérateur désigné (ou agréé) pourrait rassembler tous les
DEEE dans une plateforme de collecte construit dans le respect des normes de sécurité et d'environnement. A ce niveau, les DEEE seront catégorisés, dépollués, dépoussiérés et testés avant de procéder à leur reconditionnement ou à leur démantèlement en perspective de leur valorisation (Figure 2).

La mise en œuvre du plan d'organisation de la filière de gestion des DEEE proposée pour la gestion des composants des déchets électroniques doit être accompagnée d'une bonne sensibilisation sur les DEEE mais aussi par un encadrement juridique et institutionnel. A cet effet, une bonne politique de gestion des DEEE passera nécessairement par :

- la définition consensuelle des DEEE

- $\quad$ l'accessibilité des équipements à recycler;

- la mise en place d'une filière et d'infrastructures de gestion des DEEE ;

- la clarification des responsabilités des parties prenantes;

- $\quad$ l'application de la Responsabilité Elargie du Producteur (REP);

- la mise en place de fonds incitatifs par l'Etat ou par les Privé à travers la Responsabilité Sociétale d'Entreprise (RSE);

- le payement d'une éco-contribution par l'utilisateur (consentement);

- $\quad$ l'adoption de normes, de lois et/ou règlements nationaux spécifiques aux DEEE ;

- l'encadrement et la définition claire des mécanismes d'importation des équipements ;

- $\quad$ l'initiation de politiques nationales et régionales autour de la gestion des DEEE ;

- la coordination des actions par la mise en place d'un système d'information et d'alerte national et régionale.

Une stratégie nationale de collecte et de recyclage pourrait être inscrite comme projets pour la finance carbone et permettrait d'illustrer l'effort du Sénégal dans la préservation de l'environnement. 


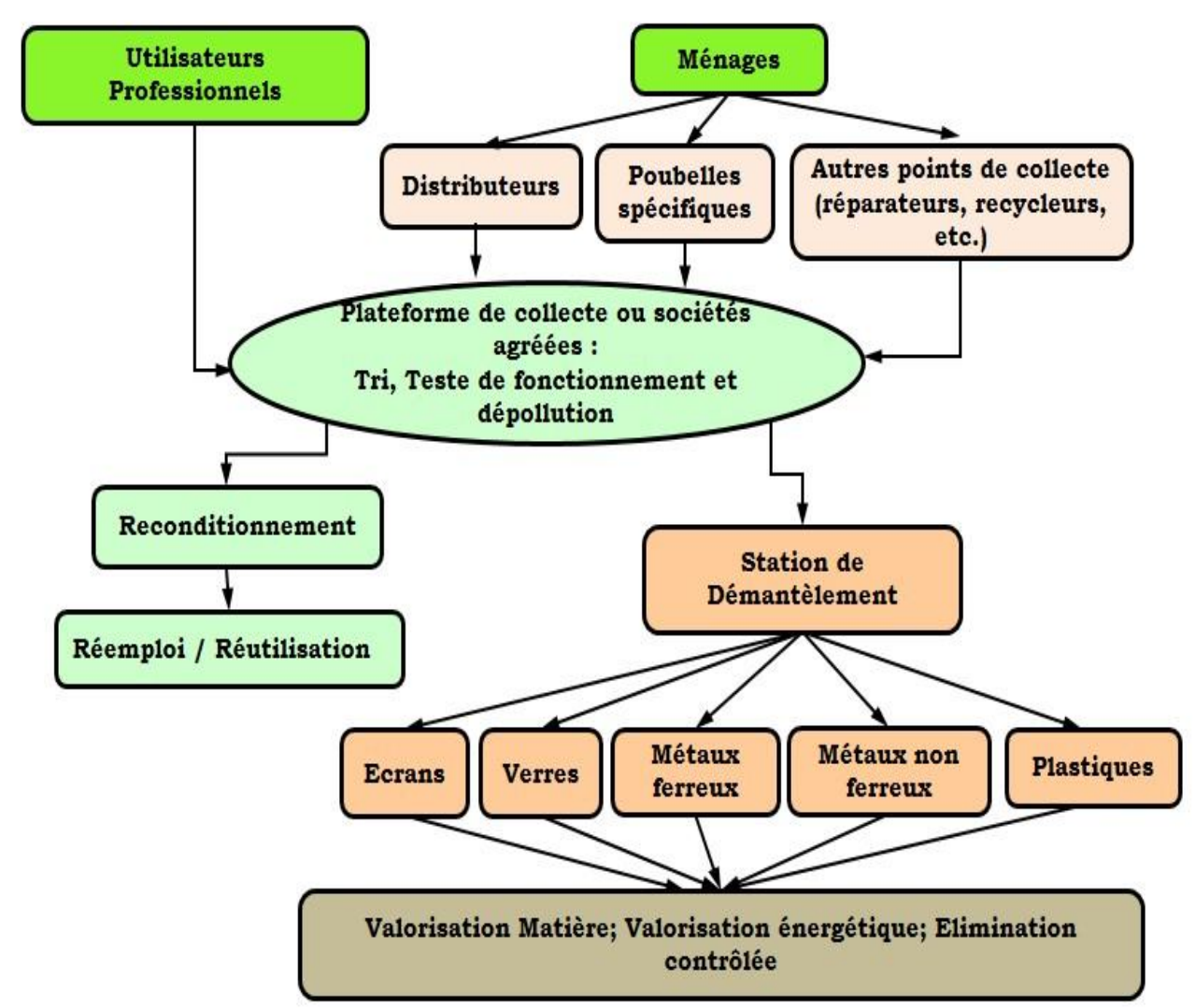

Figure 2 : Proposition d'un schéma d'organisation de la filière de gestion des DEEE au Sénégal.

\section{Conclusion}

La gestion des DEEE au Sénégal concerne beaucoup d'acteurs notamment, les importateurs, les distributeurs, les utilisateurs, les réparateurs, les recycleurs et les récupérateurs. Elle implique en majorité les jeunes et les hommes. Les interrelations désarticulées entre les acteurs montrent l'inexistence d'une filière organisée de gestion des DEEE au Sénégal. Malgré les efforts de l'Etat qui a mis en place un centre de démantèlement, le stockage de ces déchets dangereux est pratiqué dans les ménages, les services de maintenance et de réparation ainsi que les entrepôts des structures utilisant les Technologies de l'Information et de la Communication (TIC). Des pratiques informelles de recyclage sous-tendent une véritable économie circulaire qui se développe autour de la gestion de ces déchets dangereux dont les conséquences négatives sont exacerbées par le manque d'information des populations. En outre, plus de la moitié des acteurs n'a pas de connaissances sur les impacts des déchets électroniques. L'ineffectivité de la collecte sélective des déchets et le développement d'activités informelles de recyclage sont sources de pollution. Cependant, une organisation de la filière et une mise en place d'un cadre juridique spécifique aux DEEE pourraient aider à assurer durablement leur gestion écologiquement rationnelle.

\section{CONFLIT D'INTERETS}

Les auteurs déclarent n'avoir aucun conflit d'intérêts. 


\section{CONTRIBUTIONS DES AUTEURS}

La conception et la planification de l'étude ont été faites par DD, CD, EMS, JBG, MD et CIDG; l'acquisition des données, l'analyse et l'interprétation par DD ; la rédaction du manuscrit par $\mathrm{DD}, \mathrm{CD}, \mathrm{EMS}$, $\mathrm{JBG}$; la révision critique par $\mathrm{CD}$ et CIDG.

\section{REMERCIEMENTS}

Les auteurs remercient le CRDI (Centre de Recherches pour le Développement International) et Mme Ramata Molo THIOUNE pour tous les soutiens dans la réalisation de ce travail. Ils remercient le Professeur Bienvenu SAMBOU, Directeur de l'Institut des Sciences de l'Environnement pour son appui considérable à l'équipe du laboratoire.

\section{REFERENCES}

ANSD (Agence Nationale de la Statistique et de la Démographie), Sénégal. 2006. Rapport national de l'Enquête de suivi de la pauvreté au Sénégal 2005/2006.

ANSD (Agence Nationale de la Statistique et de la Démographie), Sénégal. 2009. Situation Economique et Sociale du Sénégal en 2009, 304.

ANSD (Agence Nationale de la Statistique et de la Démographie), Sénégal. 2013. http://www.recensement.sn/fr/._Consulté le 07 août 2014

Banque Mondiale. 2014. Situation socioéconomique du Sénégal, ( $1^{\text {ère }}$ édn). Apprendre du passé pour un avenir meilleur, 50 pages

Brett HR. 2009. An assessment of global production and environmental impacts. Science of the Total Environment, 408: 183-191. Doi:10.1016/j.scitotenv.2009. 09.044

Chan JKY, Wong MH. 2012. A review of environmental fate, body burdens, and human health risk assessment of PCDD/Fs at two typical electronic waste recycling sites in China. Science of the Total Environment, 463-464: 11111123. DOI: $10.1016 /$ j.scitotenv.2012.07. 098
CSE (Centre de Suivi Ecologique), Sénégal, 2010. Rapport sur l'état de l'Environnement au Sénégal, 268 pages

Diaby V, YAPO AF, ADON AM, YAPI HF, DJAMA AJ et DOSSO M. 2016. Biotoxicité hématologique du sulfate de cadmium chez les rats Wistar. Int. J. Biol. Chem. Sci., 10(4): 1765-1772. DOI: http://dx.doi.org/10.4314/ijbcs.v10i4.25

Diop C, Thioune R. 2014. Les déchets électroniques et informatiques en Afrique, défis et opportunités pour un développement durable au Bénin, au Mali et au Sénégal. Editions Karthala, $198 \mathrm{p}$.

Directives 2002/96/CE du parlement Européen et du conseil du 27 janvier 2003 relatives aux déchets d'équipements électriques électroniques (DEEE)

Donou B, ISSA MS, Eshogba-Olojoba A. 2016. Technique de traitement des déchets solides ménagers au Lieu d'Enfouissement Sanitaire (LES) de Ouessè et durabilité environnementale Int. J. Biol. Chem. Sci., 10(5). DOI: http://dx.doi.org/10.4314/ijbcs.v10i5.10

EEA (Environmental European Agency). 2003. Waste from Electrical and Electronic Equipment WEEEQuantities, Dangerous Substances and Treatment Methods. European Topic Center on Waste: Copenhagen.

ENDA (Environnement Développement Action). 2006. Etude de faisabilité pour la mise en place d'une filière intégrée de collecte, de démantèlement et de valorisation des déchets informatiques à Dakar entre partenaires d'économie sociale du nord et du sud. Dakar. 2006

Frazzoli C, Orisakwe OE, Dragone R, Mantovani A. 2009. Diagnostic health risk assessment of electronic waste on the general population in developing countries' scenarios. Environmental Impact Assessment Review 30: 388-399. DOI: 10.1016/j.eiar.2009.12.004

Kang H, Schoenung J. 2005. Electronic waste recycling: a review of U.S. infrastructure 
and technology options. Resources, Conservation and Recycling, 45(4), 368400. Doi:10.1016/j.resconrec.2005.06. 001

Kiddee P, Naidu R, Wong MH, 2013. Review Electronic waste management approaches: An overview. Waste Management 33: 1237-1250. http://dx.doi.org/10.1016/j.wasman.2013 .01 .006

Kong S, Liu H, Zeng H, Liu Y. 2012. The 7th International Conference on Waste Management and Technology The status and progress of resource utilization technology of e-waste pollution in China. Procedia Environmental Sciences, 16: 515-521. Doi: 10.1016/j.proenv.2012.10.071

OMS (Organisation Mondiale de la Santé) et Ministère de l'Ecologie et de la Protection de la Nature du Sénégal. 2008. «Projet de Dépollution du sol et désintoxication des personnes contaminées au plomb à Thiaroye Sur Mer (Ngagne diaw)». $23 \mathrm{P}$.

Pant D, Joshi D, Upreti MK, Kotnala RK. 2012. Chemical and biological extraction of metals present in $\mathrm{E}$ waste: A hybrid technology. Waste Management, 32: 979-990. DOI: 10.1016/j.wasman.2011. 12.002

Qu Y, Zhu Q, Sarkis J, Geng Y, Zhong Y. 2013. A review of developing an ewastes collection system in Dalian, China. Journal of Cleaner Production, 52: 176-184. DOI:10.1016/j.jclepro. 2013.02.013

Rotter VS, Chancerel P, Schill WP. 2011. Practicalities of individual producer responsibility under the WEEE directive: experiences in Germany. Waste Management \& Research, 29: 931-944. DOI: $10.1177 / 0734242 X 11415753$

Secrétariat de la Convention de Bâle. 2011. Rapport technique de diagnostic national des mouvements transfrontières et de la gestion des DEEE, SBC e-Waste Africa Project Benin octobre 2011, V.1.0

Some YSC, Kangambéga E, Béné A, Diendéré I. 2006. Les déterminants du choix des conteneurs plastiques dans la production des plants à Ouagadougou (Burkina Faso) : une contribution à la gestion des déchets plastiques; Int. J. Biol. Chem. Sci., 10(6): 2637-2645. DOI: http://dx.doi.org/10.4314/ijbcs.v10i6.18

UNEP (United Nations Environment Program). 2009. Recycling-from e-waste to resources: Sustainable innovation and technology. UNEP Transfer industrial sector studies. STEP-solving The Ewaste problem, July 2009.

US EPA, 2012. A dioxin science assessment consumer fact sheet February 2012. Available online at http://www.epa.gouv/ dioxin/pdfs/EPA_Dixin-factsheet2012.pdf

Wang DL, Cai ZW, Jiang GB, Leung A, Wong MH, Wong WK. 2005. Determination of polybrominated diphenyl ethers in soil and sediment from an electronic waste recycling facility. Chemosphere, 60: 810-816. https://doi.org/10.1016/j.chemosphere.20 05.04 .025

Wang HM, Yu IJ, Han M, Yang SW, Yang Y. 2009. Estimated PBDE and PBB congeners in soil from and electronic waste disposal site. Bulletin of Environmental Contamination and Toxicology, 83: 789-793. DOI: 10.1007/s00128-009-9858-6

Widmer R, Oswald-Krapf H, Sinha-Khetriwal D, Schnellmann M, Böni H. 2005. Global perspectives on e-waste. Environmental Impact Assessment Review, 25: 436-458. https://doi.org/10.1016/j.eiar.2005.04.00 $1 \mathrm{~s}$

Wone S, Rochat D. 2009. Rapport technique de l'état des lieux de la gestion des edéchets au Sénégal. SENECLIC, 53 p.

Zhao G, Dong MH, Rao K, Luo J, Wang D, Zha J, Huang S, Xu Y, Ma M. 2008. PBBs, PBDEs and PCBs levels in hair of residents around e-waste disassembly sites in Zhejiang province, China and their potential sources. Science of total Environment, 397: 46-57. DOI: 10.1016/j.scitotenv.2008.03.010. 